\title{
Effects of Reducing Agents on Rheological Properties, Inhibition of Lysinoalanine Formation, and Spinnability of Soy Protein
}

\author{
Keiko Katsuta and Isao HaYAKawA* \\ Faculty of Education, Niigata University, \\ 8050 Ninocho, Igarashi, Niigata-shi 950-21, Japan \\ *Department of Food Science \& Technology, Faculty of Agriculture, \\ Kyushu University, Hakozaki, Higashi-ku, Fukuoka 812, Japan
}

Received October 25, 1983

\begin{abstract}
The relationships among the spinnability, the rheological properties, the spun fiber strength, and the inhibition of lysinoalanine (LAL) formation with the addition of reducing agents were studied to get safe, edible, fibrous soy protein, having excellent spun fiber strength, when a dope of $20 \%$ protein concentration was used as a normal dope. It was found that cysteine and 2mercaptoethanol were effective in reducing LAL formation and the dopes prepared with the addition of these agents showed almost the same spun fiber strength as that of the normal dope prepared without agents. Especially, cysteine was the most effective agent for inhibiting LAL formation to get fibrous protein for meat analogues. The most suitable concentration for inhibiting LAL formation was $2 \%$ cysteine in the total protein. The dopes with the addition of 2mercaptoethanol and $\mathrm{Na}_{2} \mathrm{SO}_{3}$ had lower viscoelastic values and their frequency dependence of $G^{\prime}$ was higher than that of the normal dope. However, the dopes with the addition of other reducing agents ( $\mathrm{NaHSO}_{3}$, glycine, reduced glutathione) had higher viscoelastic values and lower frequency dependence of $G^{\prime}$. The viscoelastic values of the dopes with the addition of 2-mercaptoethanol, $\mathrm{Na}_{2} \mathrm{SO}_{3}$, and that of the normal dope were decreased with the lapse of time, but the dopes prepared by the addition of other agents had constant viscoelastic values.
\end{abstract}

From the general behavior of soy protein, it may be concluded that one of the most important procedures to prepare a good spinnable dope is alkaline hydrolysis of the soy protein because soy protein is globular. ${ }^{1 \sim 8)}$ Since soy protein is denatured with alkali, we explained the relationships between the spinnability and the degree of alkaline denaturation of the dope. ${ }^{\sim 4,6)}$ Effects of alkali on the gelformation mechanism of dope are also reported on the basis of rheological measurements. ${ }^{5)}$

On the other hand, a large amount of lysinoalanine (LAL) is found in the dope and in the spun fiber which have been used in our experiments. The chemical changes that produce unusual amino acids such as LAL and lanthionine during alkali treatment need to be explained and strategies to minimize or prevent these reactions need to be developed. LAL is formed from the crosslinkage reaction of $\varepsilon$-amino group of lysine with the double bond of dehydroalanine which is formed through $\beta$-elimination from cystine, serine, or serine derivatives by alkaline treatment of food protein. ${ }^{11,12)}$ In contrast, Sternberg and Kim have reported that LAL might be generated even in the absence of alkali, during conventional home-cooking or traditional cooking procedures in food, ${ }^{13)}$ therefore LAL is probably not harmful to human beings. However, until the safety and the effects of LAL on the human body are established, its formation in processed food should be limited by control of the processing conditions or by the addition of blocking agents.

In our previous paper, ${ }^{3)}$ we found that LAL formation was proportional to protein concentration in the dope, and we obtained good results in reducing LAL formation when the dope was prepared with low protein concentrations. Since the spun fiber strength dur- 
ing spinning is also proportional to the protein concentration of the dope, the lowered protein concentrates caused weak fiber. ${ }^{7)}$ Therefore, in order to obtain excellent safe spun fiber, the dope should be prepared with high protein concentrations and also LAL formation must be inhibited by another method, for example, by the application of reducing agents.

The present study was done to find the relationships between the spinnability and the rheological properties of dope which was prepared with reducing agents to inhibit LAL formation.

\section{MATERIALS AND METHODS}

1) Soy protein (Fujipro-R) was obtained from Fuji Oil Manufacturing Co., Ltd.

2) L-Cysteine, L-cystine, 2-mercaptoethanol, glutathione (reduced), glycine, sodium sulfite, sodium hydrogen sulfite and other reagents were of analytical grade and were obtained from Wako Pure Chemical Industries Co., Ltd.

3) TLC cellulose sheet No. $13255(20 \times 20 \mathrm{~cm})$ was obtained from Eastman Co., Ltd.

4) Dope was prepared using a Polytron PT- $45 / 50$ mixer (Kinematica Co., Ltd., Swizerland). A dope of $20 \%$ protein and $1.2 \% \mathrm{NaOH}$ was mixed and used as a normal dope $(\mathrm{pH} 12.65)$.

5) Presence of LAL was determined by the TLC method according to the method of Sternberg and Kim. ${ }^{14)}$ All readings of plots which were developed and colored by ninhydrin were carried out with a spectrodensitometer, Model DMU-33C, of Toyo Scientific Industry Co., Ltd. (see ref. 3).

6) Temperature during preparation of dope was measured by a $\mathrm{C}-\mathrm{C}$ thermocouple (see ref. 3 ).

7) Spinning of dope was carried out with a trial wet spinning machine designed by Hayakawa (see ref. 2).

8) Spin fiber strength during the spinning process was calculated by the drag received on a columnar body moving in the stationary fluid (see ref. 7).

9) Viscoelastic properties were measured with a 3D Rheometer from Iwamoto Seisakusho Co., Ltd., and the measurement was started $30 \mathrm{~min}$ after the dope was poured into the rheometer. Dynamic modulus $\left(G^{\prime}\right)$ and loss modulus $\left(G^{\prime \prime}\right)$ were calculated by a modified Markovitz's equation, and the data were fed into a computer, SORD M 23 Mark II, for analysis and drawing of figures.

\section{RESULTS}

Effects of reducing agents on inhibition of $L A L$ formation

This investigation is based on the works of
Friedman, ${ }^{12,16 \sim 18)}$ Finley et $a .^{11,19)}$ and Sternberg et al. ${ }^{20)}$ that thiol derivatives such as cysteine and sulfite ions decrease LAL formation during alkali treatment of several kinds of food protein.

Figure 1 shows the effects of reducing agents on LAL formation in the dope.

The original sample (Fujipro-R) contains $0.09 \mathrm{mg} \mathrm{LAL} / \mathrm{g}$ protein, and the normal dope contains $1 \mathrm{mg} \mathrm{LAL} / \mathrm{g}$ protein. But LAL formation in the normal dope was prevented by the addition of $2 \sim 4 \%$ reducing agents (agent/protein). Especially, thiol derivatives such as cysteine, mercaptoethanol, and glutathione reduced the LAL formation to less than $30 \%$ of the normal dope.

While it is interesting that cystine was effective in preventing LAL formation, Friedman reported that LAL formation was stimulated by the addition of cystine, because dehydroalanine should be increased by $\beta$-elimination of cystine. We expected to have the same results as he had, we found that but opposite. We proceeded with the study in oder to find reason why our results were different from the results of Friedman et al.

Figure 2 showed the temperature-rising

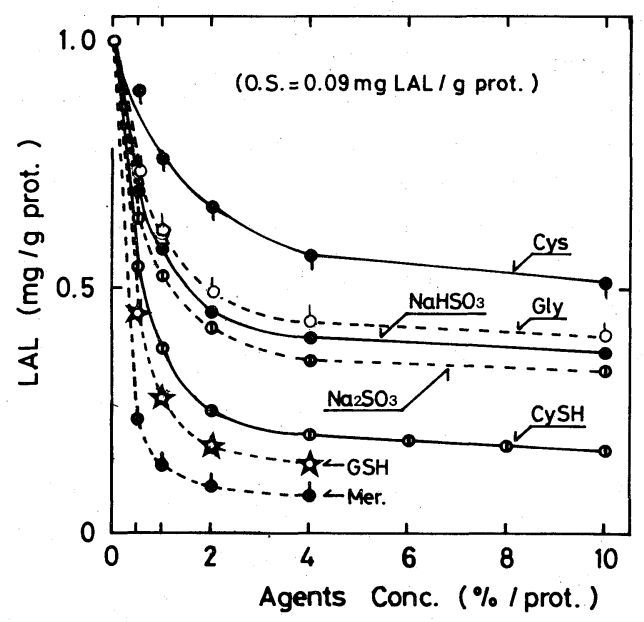

FIG. 1. Effects of Reducing Agents on Inhibition of LAL Formation in the $20 \%$ Protein Dope.

O. S., in Fig. 1, shows an original sample, i.e. a commercial isolated soy protein. The dope prepared with $20 \%$ protein concentrate and $1.2 \% \mathrm{NaOH}$ was used as a normal dope. This dope contained $1.0 \mathrm{mg}$ LAL per gram of protein. 


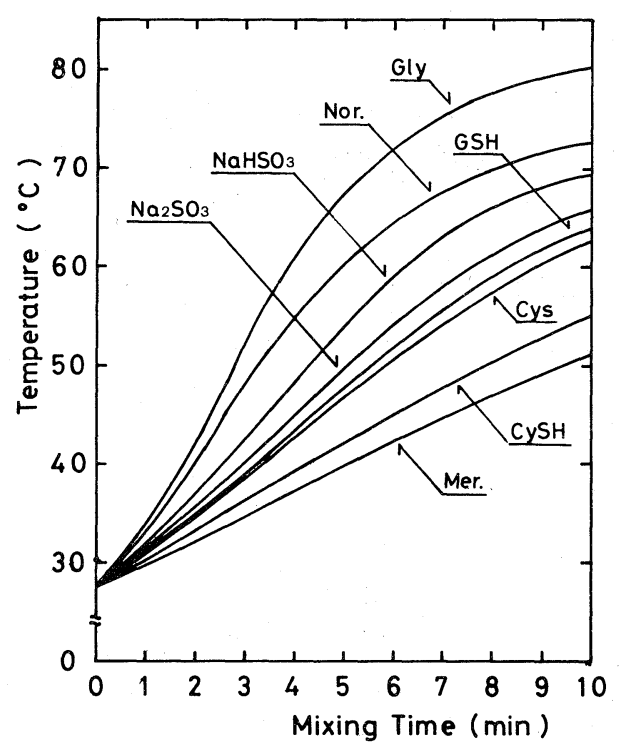

FIG. 2. Effects of Reducing Agents on Temperature Rise as Dope is mixed:

The dope is the same as in Fig. 1. Each addition of Agents is $2 \%$ of total protein.

curves of dope when the dope was mixed with the addition of $2 \%$ reducing agent (agent/protein). Since frictional heat was generated by mixing of the dope, the temperature of the dope rose with mixing time. It was found that the temperature of all dopes except the dope with glycine added was lower than that of the normal dope. The dope with cystine added also reduced the temperature rise, but it was not so effective as cysteine and mercaptoethanol. The results suggest that not only the reaction mechanism of the $\beta$-elimination under alkaline conditions, but also the mechanism of reaction by heating the protein is concerned with LAL formation in dope. Therefore, the latter of the two reaction mechanisms of LAL formation could be prevented by the addition of cystine.

\section{Effects of reducing agents on spun fiber strength} of soy protein dope

The effects of reducing agents on LAL formation and on spun fiber strength were studied, and the results are shown in Table I.

From these results, the dope prepared with the addition of cysteine or mercaptoethanol
Table I. Effects of Reducing Agents ON LAL CONTENTS AND STRENGTH OF SPUN FibER

\begin{tabular}{lcc}
\hline & $\begin{array}{c}\text { LAL Contents } \\
(\mathrm{mg} / \mathrm{g} \text { prot. })\end{array}$ & $\begin{array}{c}\text { Strength } \\
\text { (dyn) }\end{array}$ \\
\hline $\begin{array}{l}\text { Control }(1.2 \% \\
\mathrm{NaOH})\end{array}$ & 1.35 & 1.61 \\
$\begin{array}{l}\text { Cysteine } \\
\text { Glutathione reduced }\end{array}$ & 0.31 & 1.53 \\
Mercaptoethanol & 0.27 & 0.74 \\
Glycine & 0.14 & 1.66 \\
$\mathrm{Na}_{2} \mathrm{SO}_{3}$ & 0.64 & - \\
$\mathrm{NaHSO}_{3}$ & 0.63 & 1.10 \\
$\mathrm{Cystine}^{2 \%}$ Agents/prot. & 0.63 & 0.69 \\
\hline
\end{tabular}

showed as a good spun fiber strength as that of the normal dope. The fiber strength of the dope which was prepared with the addition of $\mathrm{NaHSO}_{3}$ or glutathione was decreased to about half the strength of the normal sample, and the dope prepared with the addition of glycine or cystine had no spinnability. Therefore, mercaptoethanol and cysteine were good agents to prevent LAL formation. As it is undesirable to use mercaptoethanol for safety reason, cysteine is the best agent. Then the relationships between the effects of cysteine on the prevention of LAL formation and on the spun fiber strength were studied. The results are shown in Fig. 3.

The spun fiber strength was decreased as the amount of added cysteine increased. The dope could not be spun when the addition of cysteine exceeded $6 \%$ of the total protein. From the above results, the prevention of LAL formation was sufficient with the addition of $2 \%$ cysteine.

Effects of reducing agents on viscoelastic behavior

As shown in Table I, changes in spun fiber strength had appeared when the dope was prepared with the addition of reducing agents. Since that may reflect changes of the viscoelastic behavior of the dope, dynamic viscoelastic properties of the dope were measured. The results are illustrated in Fig. 4. 


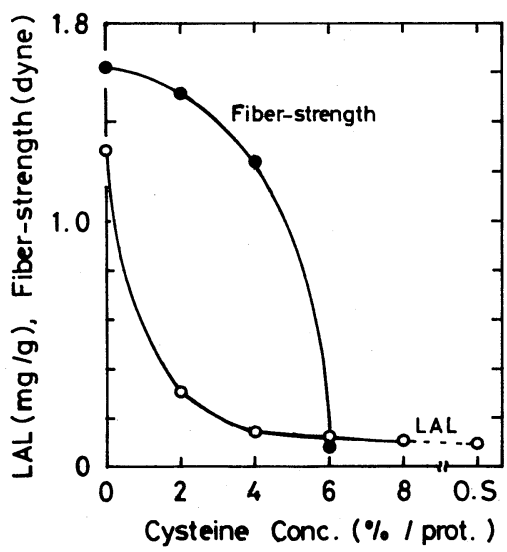

FIG. 3. Effects of Cysteine on Inhibition of LAL Formation in the Dope and on the Strength of Spun Fiber. O. S. and the normal dope are the same as in Fig. 1.

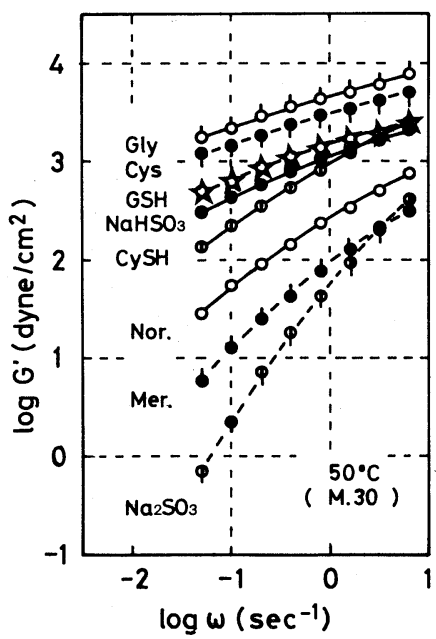

FIG. 4. Effects of Reducing Agents on $G^{\prime}$ Curve.

The dopes have the same components as in Fig. 3. The viscoelastic value was measured at $50^{\circ} \mathrm{C}$.

As compared with the normal dope, the dopes prepared with the addition of $\mathrm{Na}_{2} \mathrm{SO}_{3}$ or mercaptoethanol have lower $G^{\prime}$ values, but the above two dopes have a higher frequency dependence of $G^{\prime}$. On the other hand, the dopes prepared with the addition of the other agents showed higher viscoelastic values than that of the normal dope, and those dopes have lower frequency dependence of $G^{\prime}$, except the dope with the addition of cysteine. The dope prepared with the addition of cysteine had a higher viscoelastic value than that of

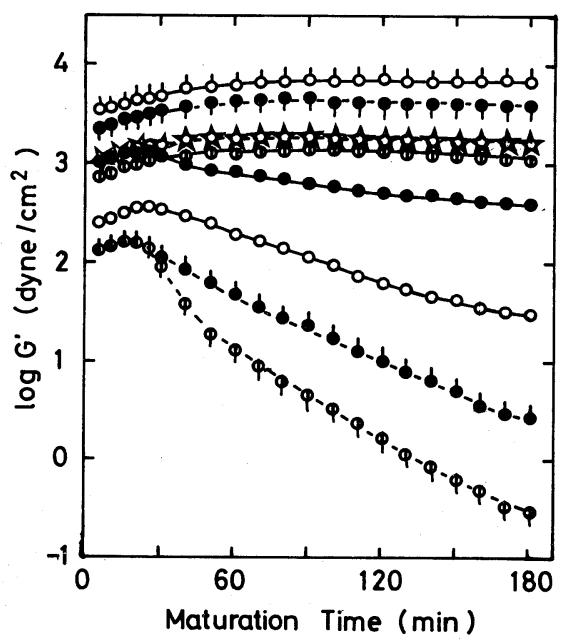

FIG. 5. Effects of Reducing Agents on the Change of $G^{\prime}$ Value during Dope Maturation.

The dopes have the same components as in Fig. 2, and each symbol in this figure is the same as that in Fig. 4. The viscoelastic values are measured at $50^{\circ} \mathrm{C}, 0.25 \mathrm{~Hz}$.

the normal dope, but its frequency dependence of $G^{\prime}$ showed the same behavior as that of the normal dope. Further, the effects of maturation time on the viscoelasticity of the dope are shown in Fig. 5, because the viscoelasticity of the dope changes with time.

The viscoelastic value of the normal dope and the dope prepared with the addition of mercaptoethanol or $\mathrm{Na}_{2} \mathrm{SO}_{3}$ decreased continuously after $30 \mathrm{~min}$ of maturation. This shows that protein in the dope is first unfolded and then is hydrolyzed into small molecules by alkali. Since mercaptoethanol denatures the protein, especially by cleaving the $\mathrm{S}-\mathrm{S}$ bonds, the intermolecular crosslinkage of soy protein in the dope is amputated, and the gel-network structure of the dope disintegrates. Accordingly the viscoelastic value of the dope rapidly decreased upon the addition of mercaptoethanol. In the case of the dope prepared by the addition of $\mathrm{Na}_{2} \mathrm{SO}_{3}$, since this agent has an alkaline effect like $\mathrm{NaOH}$, the dope is exposed to excessively alkaline conditions. The vigorous alkaline treatment caused the breakdown of protein molecules in the dope.

On the other hand, the viscoelastic value of 


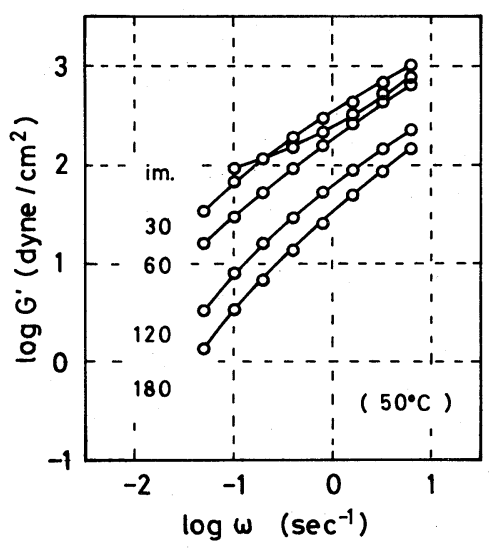

FIG. 6. Effects of Maturation Time on the Frequency Dependence of Normal Dope.

im. shows non-matured dope, and other numbers show maturation time.

dopes prepared with the addition of the other agents were constant after $30 \mathrm{~min}$ of maturation, and also equilibrated for $3 \mathrm{hr}$ except the dope prepared by the addition of $\mathrm{NaHSO}_{3}$. The present results suggest that these dopes have rigid and stable structures of gel network.

Viscoelastic values of all dopes increased the initial value up to $30 \mathrm{~min}$ because the inner temperature of the dope should have dropped to $50^{\circ} \mathrm{C}$, and the memory of deformation when the dope was poured into the rheometer should have been relaxed after $30 \mathrm{~min}$. Therefore, dynamic viscoelastic behavior of the normal dope was measured with various maturation times, and the results are illustrated in Fig. 6. The $G^{\prime}$ curve measured immediately (im. in Fig. 6) after being poured into the rheometer was different from the other curves at the low frequency side. If the dope could be controlled at $50^{\circ} \mathrm{C}$ immediately after being poured into the rheometer and also strain (the memory of deformation) in the dope could be relaxed soon, the curve (im.) in Fig. 6 would have been shifted to a little higher position than that of the dope measured after $30 \mathrm{~min}$ of maturation. The $G^{\prime}$ curve would also have showed the sharp "terminal region" parallel with the other curves.

\section{DISCUSSION}

Though LAL has induced nephrotoxity in rats, it is probably not harmful to humans, ${ }^{9,10)}$ because it is formed under conventional cooking conditions and it appears to be ubiquitous. Nevertheless, since LAL inflicts a loss of available lysine on food and its physiological effects on the human body have not been elucidated, we must be careful to prevent its formation in processed foods by controlling the processing conditions or adding blocking agents, nucleophiles such as thiols or sulfite ions.

We has already investigated how to control LAL formation in the preparation of dope, and one of the good methods was to decrease the protein concentrates in the dope. However, the dopes of low protein concentrates produced weak spun fibers, and probably would be difficult to apply to the present industrial process.

In this study, protein concentrates are held at the most suitable concentration $(20 \%)$ in order to obtain the best spun fiber strength, and then the inhibition of LAL formation was tried by the addition of reducing agents to the dope, according to Friedman's work. ${ }^{18)}$ Friedman said that nucleophiles can inhibit LAL formation by at least three mechanisms. First, by direct competition, the added nucleophile can trap dehydroalanine to form their respective adducts such as lanthionine. Second, the added nucleophile can cleave S-S bonds and thus generate free $\mathrm{SH}$ groups, which can react with dehydroalanine. LAL formation is inhibited by indirect competition of SH groups for dehydroalanine. Third, the added nucleophile, by cleaving disulfite bonds, can diminish a potential source of dehydroalanine, since the resulting cysteine residues would be expected to undergo the reaction of elimination to form dehydroalanine much less readily than the original cystine (disulfite) residues.

We found that LAL formation in the dope occurred under two conditions; one was alkaline treatment, and other was frictional heat which was generated while the dope was 
mixed. All reducing agents except glycine reduced the generation of frictional heat. Therefore, even cystine could reduced the LAL formation, contrary to Friedman's finding. ${ }^{18)}$ Besides, the addition of mercaptide was more effective than that of sulfite in inhibiting LAL formation, since thiol groups would strongly prevent $\mathrm{LAL}$ formation by competition or suppression of dehydroalanine and by reducing the frictional heat.

While the dopes prepared by the additions of mercaptoethanol and cysteine showed almost the same spun fiber strength as that of the normal dope, the dopes prepared by the addition of other agents reduced the spun fiber strength. Mercaptoethanol was the most effective agent for the inhibition of LAL formation and on the spun fiber strength. However, considering the safety of the processed food, the addition of cysteine is the best method.

When the agents were added to the dope, the viscoelastic properties of the dope produced could be classified into two groups. One group had an increase of the frequency dependence of $G^{\prime}$ and continuous decrease of the viscoelastic values with the lapse of maturation time as compared with that of the normal dope. The other had a decrease of frequency dependence of $G^{\prime}$, but the viscoelastic value was stabilized. Mercaptoethanol and $\mathrm{Na}_{2} \mathrm{SO}_{3}$ belonged to the former group, and the other agents (glycine, glutatione, $\mathrm{NaHSO}_{3}$, and cystine) belonged to the latter group. The dope prepared with the addition of cysteine showed the same frequency dependence as that of the normal dope, but cysteine looked like the latter group.

From the results of Table I, Figs 4 and 5, at least two things can be assumed. First, the spinnability and/or the spun fiber strength of dope would be reduced when the viscoelastic values of dope increased or decreased excessively. This assumption suggests that the dynamic viscoelastic behavior of dope has a critical zone. In a previous paper, ${ }^{8)}$ we had announced the critical zone of spinnable dope. Second, the addition of a few kinds of agents would cause excessive crosslinkage in the dope and thus a very rigid gel-network structure would be formed. Because the dopes prepared by the addition of glycine and cystine could not be extruded from a spinnaret, and so had no spinnability.

Finally, it was interesting that the dope prepared by the addition of reducing agents can form a stable and the rigid gel structure in the dope, except for $\mathrm{Na}_{2} \mathrm{SO}_{3}$ and mercaptoethanol. We proceed to elucidate the gelforming mechanism of soy protein in our following study.

\section{REFERENCES}

1) I. Hayakawa and K. Katsuta, Nippon Shokuhin Kogyo Gakkaishi, 28, 347 (1981).

2) K. Katsuta, I. Hayakawa and D. Nomura, Nippon Nôgeikagaku Kaishi, 56, 189 (1982).

3) K. Katsuta, I. Hayakawa and D. Nomura, Nippon Nôgeikagaku Kaishi, 56, 435 (1982).

4) I. Hayakawa, K. Katsuta and S. Kawasaki, Agric. Biol. Chem., 46, 1997 (1982)

5) K. Katsuta and I. Hayakawa, Nippon Shokuhin Kogyo Gakkaishi, 30, 20 (1983).

6) K. Katsuta and I. Hayakawa, Nippon Nôgeikagaku Kaishi, 56, 1143 (1982).

7) K. Katsuta and I. Hayakawa, Nippon Shokuhin Kogyo Gakkaishi, 30, 264 (1983).

8) K. Katsuta and I. Hayakawa, Agric. Biol. Chem., 48 , 727 (1984).

9) A. P. de Groot and P. Slump, J. Nutr., 98, 45 (1969).

10) J. C. Woodard, D. D. Short, M. R. Alvarez and J. P. Reyniers, "Protein Nutritional Quality of Foods and Feeds," ed. by M. Friedman, Marcel Dekker, New York, 1975, p. 595.

11) J. W. Finley, J. T. Snow, P. H. Johnston and M. Friedman, J. Food Science, 43, 619 (1978).

12) M. Friedman, "Functionality and Protein Structure," ACS Symposium Series 92, ed. by Am. Chem. Soc., 1979, pp. 225 235.

13) M. Sternberg, C. Y. Kim and F. J. Schwonde, Science, 190, 992 (1975).

14) M. Sternberg and C. Y. Kim, J. Food Sci., 40, 1169 (1975).

15) Koubunshi Gakkai, "Rheology Measurement" (in Japanese), ed. by Kubunshi Gakkai, Kyoritsu Syuppan Co., Tokyo, 1965, pp. 56 59.

16) M. Friedman, "Nutritional and Medical Consequence," ed. by M. Friedman, Plenum Press, New York, 1977, pp. $1 \sim 27$.

17) M. Friedman, "Food Proteins," ed. by J. R. Whitaker and R. R. Tannenbaum, AVI Publishing, Westport, Connecticut, 1977, pp. 446 483.

18) M. Friedman, "Nutritional Improvement of Food and Feed Proteins," ed. by M. Friedman, Plenum 
Press, New York, 1978, pp. 613 648.

19) J. W. Finley, J. T. Snow, P. H. Johnston and M. Friedman, "Nutritional and Medical Consequence," ed. by M. Friedman, Plenum Press, New York, 1977, pp. $123 \sim 130$.

20) M. Sternberg and C. Y. Kim, "Nutritional and Medical Consequence," ed. by M. Friedman, Plenum Press, New York, 1977, pp. 73 84 . 